
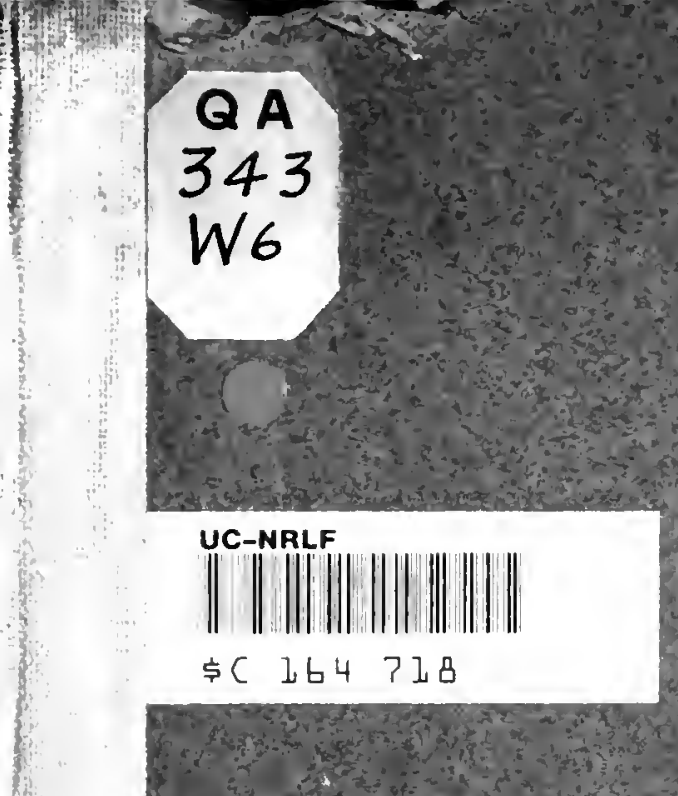


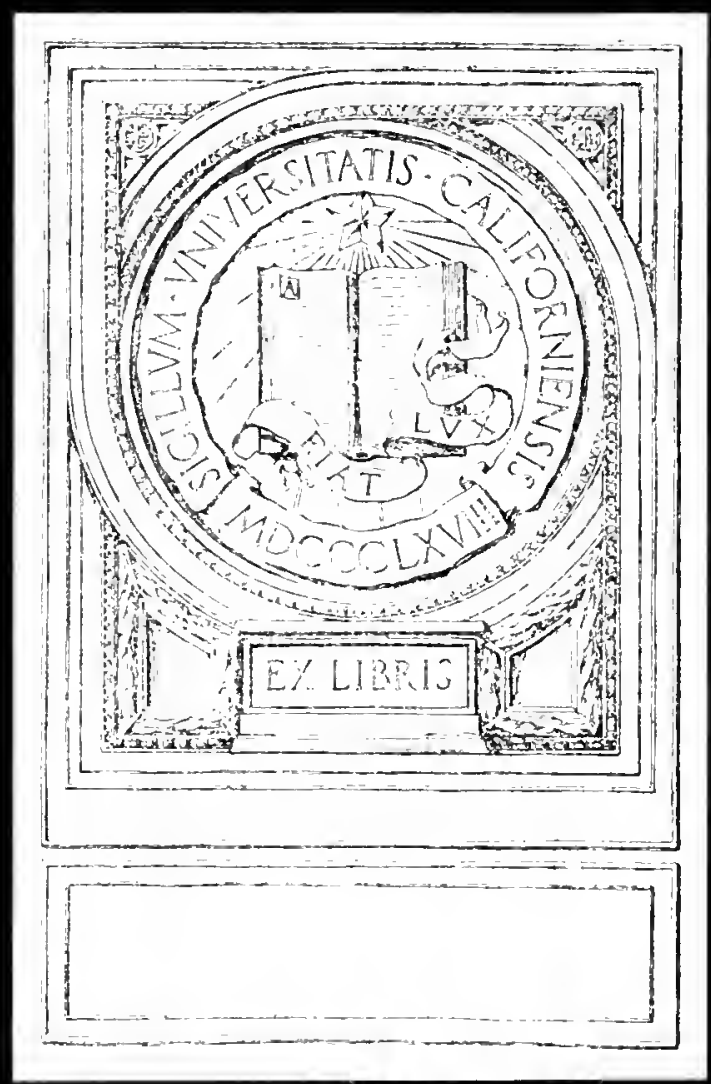




\title{
THE ELLIPTIC MODULAR FUNCTIONS ASSOCIATED WITH THE ELLIPTIC NORM CURVE $E^{7}$
}

\author{
BY \\ ROSCOE WOODS \\ A. B. Georgetown Collegge, 1914 \\ A. M. University of Maine, 1916 \\ $\overline{-1}$ \\ THESIS \\ Submitted in Partial Fulfillment of the Requirenents for the \\ Degree of \\ Doctor of Philosophy \\ ix mathenatics \\ IN \\ THE GRADUATE SCHOOL \\ OF THE \\ UNIVERSTTY OF ILLINOIS \\ 1920
}

1107 3. iy

Reprinted from the Transactions of The American Mathematical Societr, Vol. 23, No. 2, March, 1922. 


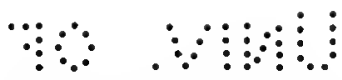

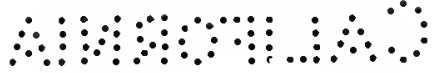




\title{
THE ELLIPTIC MODULAR FUNCTIONS ASSOCIATED WITH THE ELLIPTIC NORM CURVE $\mathbf{E}^{7}$
}

\author{
BY \\ ROSCOE WOODS \\ A. B. Georgetown College, 1914 \\ A. M. University of Maine, 1916 \\ THESIS \\ Submitted in Partil Fulfilluent of the Requirements for the \\ Degree of \\ Doctor of Philosophy \\ in MATHeMatics \\ IN \\ THE GRADUATE SCHOOL \\ OF THE \\ UNIVERSity of Illinols \\ 1920
}

Reprinted from the Transactions of the American Mathematical Society, Vol. 23, No. 2, Mareh, 1922. 

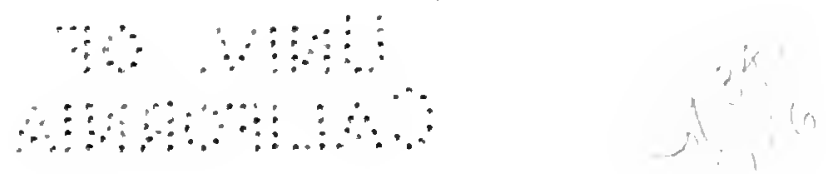

N 


\section{ACKNOWLEDGMENT}

To Professor A. B. Coble under whose supervision this paper has been written I am especially indebted for his very valuable suggestions, his deep interest and his unfailing kindness and encouragement.

UNIVERSity OF ILLINOIS, MAY, 1920.

Roscoe Woons. 


\section{CONTEN'TS}

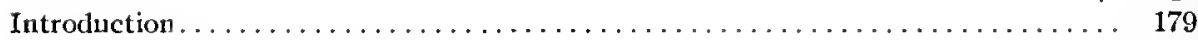

I. The groups connected with $\mathrm{E}^{7}$

1. The group $\mathrm{G}_{2.7^{2}}$ of collineations of the $\mathrm{E}^{\boldsymbol{\gamma}}$ into itself $\ldots \ldots \ldots \ldots \ldots \ldots \ldots \ldots$

2. The fixed heptahedra of the 8 cyclic $G_{7}$ 's $\ldots \ldots \ldots \ldots \ldots \ldots \ldots \ldots \ldots \ldots$

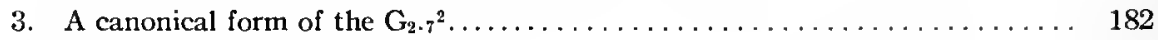

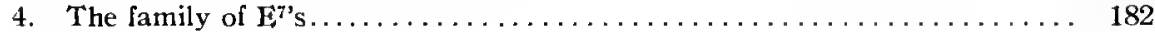

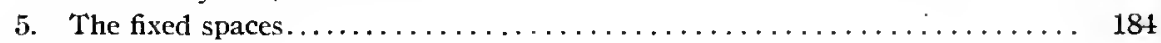

II. The quadrics on $\mathrm{E}^{7}$

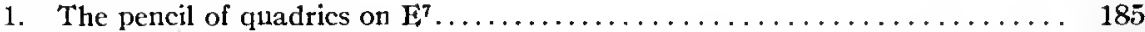

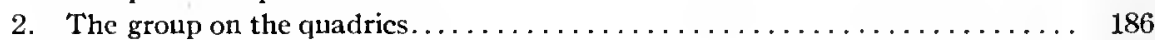

3. A Kleinian form..$\ldots \ldots \ldots \ldots \ldots \ldots \ldots \ldots \ldots \ldots \ldots \ldots \ldots \ldots \ldots \ldots \ldots$

III. The interpretation of the form $\mathrm{F}^{\prime}$

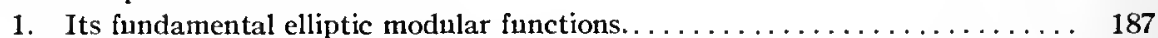

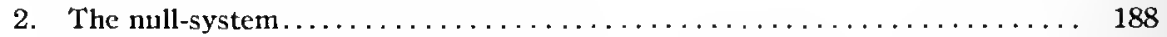

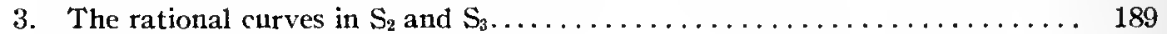

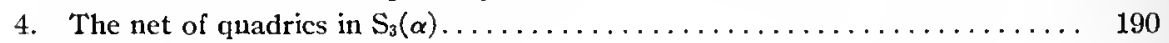

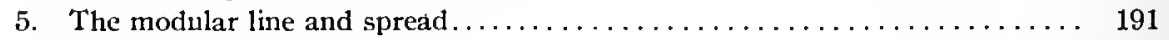

IV. The loci in $\mathrm{S}_{3}$

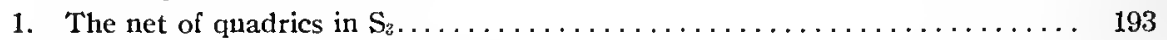

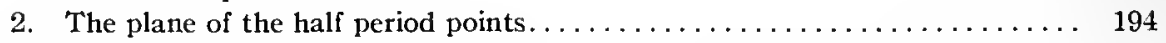

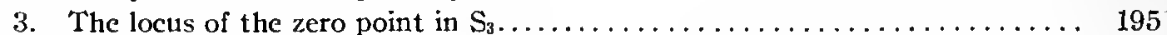

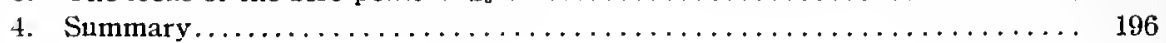




\title{
THE ELLIPTIC MODULAR FUNCTIONS ASSOCIATED WITH THE ELLIPTIC NORM CURVE $\mathrm{E}^{7 *}$
}

\author{
BY \\ ROSCOE WOODS \\ INTRODUCTION
}

The elliptic norm curve $E^{n}$ in space $S_{n-1}$ admits a group $G_{2 n 2}$ of collineations and there is a single infinity of such curves which admit the same group. A particular $E^{n}$ of the family is distinguished by the coördinates of a point on a modular curve, the ratios of these coördinates being elliptic modular functions defined by the modular group congruent to identity $(\bmod n)$. In the group $G_{\Sigma_{n^{2}}}$ there are certain involutory collineations with two'fixed spaces. If $E^{n}$ is projected from one fixed space upon the other, a family of rational curves $C^{m}$ mapping the family of $\mathrm{E}^{n \text { 's }} \mathrm{s}$ is obtained. The quadratic irrationality separating involutory pairs on $E^{n}$ involves the coördinates of a point on the modular curve and the parameter $t$ on a member of the family $C^{m}$.

Miss B. I. Miller $\nmid$ has discussed the elliptic norm curves for which $n=3,4$, 5 . In these cases the genus of the modular group is zero and a point of the modular curve can be denoted by a value of the binary parameter $\tau$. The irrationality separating involutory pairs on $E^{n}$ was used by her to define an elliptic parameter

$$
u=\int \frac{(t d t)}{\sqrt{(t \tau) \alpha_{\tau}^{\gamma-3} \alpha_{t}^{3}}}
$$

where $\alpha{ }_{\tau}^{r}$ is the tetrahedral, octahedral, or icosahedral form. This form of $u$ is invariant under all the cogredient tranformations of $t$ and $\tau$ which leave $\alpha_{\tau}^{r}$ unaltered.

The cases considered by Dr. Miller are relatively simple, due to the fact that the genus of the modular group is zero. In this paper, the case $n=7$ for which the genus is 3 , one which is fairly typical of the general case, is subjected to a

* Presented to the Society, April 14, 1922.

† See these Transactions, vol. 17 (1916), p. 259. 
similar investigation. Many of the results may be cxtended to the case where $n$ is any prime number and in some features to the case where $n$ is any odd number. By methods of geometry and group theory, we derive in this discussion the well known elliptic modular functions at tached to this group as well as some new ones and obtain a number of their algebraic properties.* This treatment suggests a number of "root functions," i. e., square roots of modular functions which are themselves uniform.

In $\S I$, the groups and subgroups associated with the $E^{7}$ are discussed and thrown into a canonical form. The equations of the transformation from $S_{6}$ to the fixed spaces $S_{2}, S_{3}$, and the equations of the groups of transformations in these spaces are derived. These have been found without the aid of function theory and have been checked with Klein's results in Klein-Fricke's Elliptische Modulfunktionen. In $\S I I$, a single Kleinian form $\dagger$ is derived which furnishes the fourteen linearly independent quadrics whose complete intersection is $E^{7}$. From this form in $\S I I I$ the fundamental elliptic modular functions $t_{1}: t_{2}: t_{4}$ are determined. Also the families $C^{2}, C^{3}$ of rational curves in $S_{2}$ and $S_{3}$ are found. In $\S I V$, the loci in $S_{3}$ are discussed. The paper closes with a parametric representation of $E^{?}$.

\section{I. 'THE GROUPS CONNECTED WITH $E^{7}$}

1. The group $G_{2 \cdot 7^{2}}$ of collineations of $E^{7}$ into itself. The homogeneous coördinates of a point of the elliptic norm curve $E^{7}$ are $x_{0}: x_{1}: \ldots: x_{6}=1: p(u)$ : $p^{\prime}(u): \cdots: p^{v}(u)$. As $u$ runs over the period parallelogram $\omega_{1}$, $\omega_{2}$ the $E^{7}$ is obtained in a six-dimensional space $S_{6}$. It is known $\ddagger$ that the only birational transformations of the general elliptic curve into itself are given by $u^{\prime}= \pm \cdot u$ $+b$, where $b$ is any constant. $\$$ From the parametric representation of the $E^{7}$ as set forth above, it is evident that seven points of the $E^{7}$ on a hyperplane section are characterized by the fact that the sum of their parameters is congruent to zero $\left(\bmod \omega_{1}, \omega_{2}\right)$ and conversely. In view of this, all transformations for which $7 b \equiv 0\left(\bmod \omega_{1}, \omega_{2}\right)$ are collineations. This congruence has three irreducible solutions

$$
b=0, b=\omega_{1} / 7, b=\omega_{2} / 7
$$

* In the case $n=4$, Miss Miller has expressed the opinion that the properties of the elliptic integral associated with $E^{4}$ and the Dyck quartic should apply to Klein's quartic which occurs in this case. This has not been verified.

$\dagger$ By a Kleinian form is meant a form in several variables invariant under isomorphic linear groups on these variables.

$\ddagger$ Appell-Goursat, Fonctions Algébriques, p. 474.

Segre, M a th e mat is che Annalen, vol. $27(1887)$, p. 296.

Klein-Fricke, Theorie der elliptischen Modulfunktionen, vol. 2, p. 241. Hereafter the initials K. F. will be used to refer to this work. 
These furnish the substitutions

$$
\begin{array}{ll}
S_{01}: u^{\prime}=u+\omega_{01}, & \\
S_{10}: u^{\prime}=u+\omega_{10}, & \omega_{i j}=i \omega_{1} / 7+j \omega_{2} / 7, \\
V: u^{\prime}=-u, & (i, j=0,1, \ldots,(0) .
\end{array}
$$

$S_{01}$ and $S_{10}$ are collineations of period seven and generate a group $G_{7^{2}}$ which is abelian in its elements. $\quad V$ is a collineation of period two which adjoined to $G_{7}$ generates a group $G_{2 \cdot 7^{2}}$. This group $G_{2 \cdot 7^{2}}$ of collineations contains all the collineations of the general $E^{7}$ into itself.

The $G_{7^{2}}$ in the $G_{2 \cdot 7^{2}}$ contains 8 cyclic $G_{7}$ 's and no other subgroups. These are denoted by $G_{\infty}, G_{1}, \ldots, G_{6}$ where $G_{\infty}$ is generated by $S_{01}$ and $G_{i}$ by $S_{10} S_{01}^{i}(i=0$, $1, \ldots, 6)$. The elements of $G_{2 \cdot 7^{2}}$ not in $G_{7^{2}}$ are of the form

$$
V_{i j}: u^{\prime}=-u+\omega_{i j} \quad(i, j=0,1, \ldots, 6),
$$

and are of period two. The $V_{i j}$ form a conjugate set. Any cyclic $G_{7}$ with one involution generates a dihedral $G_{2 \cdot 7}$ which contains seven involutions. Hence there are 56 dihedral $G$ 's. 'These with the cyclic $G_{2}$ 's complete the subgroups of $G_{2 \cdot 7^{2}}$. The relations satisfied by the generators of $G_{2 \cdot 7^{2}}$ are

$$
\begin{aligned}
& S_{01}^{7}=S_{10}^{7}=1, \\
& S_{01} S_{10}=S_{10} S_{01}, V S_{10}=S_{10}^{1} V, \quad V S_{01}=S_{01}^{-1} V .
\end{aligned}
$$

2. The fixed heptahedra of the 8 cyclic $G_{7}$ 's. The condition that a hyperplane section touch the $E^{7}$ in seven coincident points is given by

$$
7 u \equiv 0\left(\bmod \omega_{1}, \omega_{2}\right) \text {. }
$$

The irreducible solutions of this congruence furnish the 49 parameters $\omega_{i j}$ of the singular points. Under $G_{\infty}$ the 49 points $\omega_{i j}$ separate into 7 sets of seven conjugate points such that each set is on a hyperplane. Such a set of seven hyperplanes will be called a heptahedron. Since there are 8 cyclic $G_{7}$ 's, there are 8 heptahedra which will be designated by $H_{\infty}, H_{0}, \ldots, H_{6}{ }^{*}$

The 49 singular points are now arranged in a matrix (using only the subscripts) in such a way that the rows furnish the 7 sets of conjugate points which determine the 7 hyperplanes of $H_{\infty}$, while the columns furnish the 7 hyperplanes of $H_{0}$.

* The reason for calling one heptahedron $H_{\nsim}$ will appear later. These heptahedra can be determined from the resolvent equation of the 8 th degree associated with the Galois problem of degree 168. Compare K. F., vol. 1, p. 732. 


\begin{tabular}{||lllllll}
00 & 01 & 02 & .03 & 04 & 05 & 06 \\
10 & 11 & 12 & 13 & 14 & 15 & 16 \\
20 & 21 & 22 & 23 & 24 & 25 & 26 \\
30 & 31 & 32 & 33 & 34 & 35 & 36 \\
40 & 41 & 42 & 43 & 44 & 45 & 46 \\
50 & 51 & 52 & 53 & 54 & 55 & 56 \\
60 & 61 & 62 & 63 & 64 & 65 & 66
\end{tabular}$\|$

Each row of this matrix is transformed into itself by $G_{\infty}$, each column into itself by $G_{0}$. Further the seven hyperplanes of each heptahedron are linearly independent. Let us prove this for $G_{\infty}$. If the seven hyperplanes $X_{i}$ are not independent, there is a relation among them involving $7-k$ of these $X$ 's such that these $7-k X$ 's do not satisfy further relations. Then the $7-k X$ 's in this relation are all fixed under $G_{\infty}$ and meet in an $S_{k}$ which is also fixed under $G_{\infty}$. Hence $G_{\infty}$ permutes the $S_{5}$ 's on the fixed $S_{k}$ in such a way that $7-k$ of the $S_{5}$ 's are fixed. Therefore by projection from $S_{k}$ upon an $S_{5-k}$, we should have in $S_{5-k} 7-k$ fixed spaces no. $6-k$ of which were related. But such a collineation is the identity in $S_{5-k}$. Hence every $S_{5}$ on $S_{k}$ is fixed, contrary to the fact that $G_{\infty}$ has only a finite number 7 of fixed spaces.

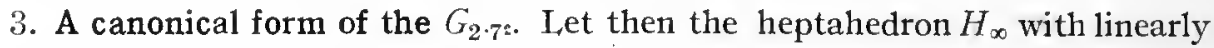
independent faces be chosen as a reference figure and denote these faces by $X_{i}$ $(i=0,1, \ldots, 6){ }^{*}$ These are determined by the rows of the matrix $(6)$. The reference figure is completed by choosing a unit-hyperplane. This hyperplane will be chosen as the one containing the singular points of the first column of the matrix (6).

In terms of the coördinates thus defined the generators of the $G_{2 \cdot 7^{2}}$ of collineations of the $E^{7}$ into itself have the form.

$$
\begin{aligned}
& S_{01}: X_{i}^{\prime}=X_{i-1} \quad\left(X_{7+i} \equiv X_{i}\right) \\
& S_{10}: X_{i}^{\prime}=\epsilon^{i} X_{i} \quad(i=0,1, \ldots, 6) \\
& V: X_{i}^{\prime}=X_{-i} \quad\left(X_{-i} \equiv X_{7-i}\right)
\end{aligned}
$$

where $\epsilon$ is a seventh root of unity. The formulas (7) constitute a first canonical form of $G_{2 \cdot 7^{2}}$.

4. The family of $E^{7}$ 's. The curve $E^{7}$ depends upon the ratio $\omega=\omega_{1} / \omega_{2}$. For each value of $\omega$, there is an $E^{7}$, hence there is a family $F$ of $E^{7}$ 's. But the

* $X_{i}$ is written instead of $X_{i}(u)$. The $X_{i}$ can be represented as the products of sigma functions, i. e.,

$$
X_{i}(u)=\alpha i \prod_{j=0}^{j=6} \sigma\left(u-\omega_{i j}\right)
$$

where the $\alpha_{i}$ are constants which insure the double periodicity of the ratios $X_{i}$. Compare $\mathrm{K}$. F., vol. 2, p. 238. 
group $G_{2 \cdot 7}$ of collineations is the same for each member of the family $F$ since its coefficients are numbers independent of the ratio $\omega$. For each curve of $F$ the set of $S$ heptahedra is the same, since the heptahedra are determined by their common $G_{2 \cdot 7^{2}}$

All collineations which leave each member of $F$ unaltered have been determined. If there are further collineations which interchange the members of $F$, they must arise from integer period transformations of determinant +1 . Consider then the transformation

$$
\begin{aligned}
& \omega_{1}^{\prime}=\alpha \omega_{1}+\beta \omega_{2} \\
& \omega_{2}^{\prime}=\gamma \omega_{1}+\delta \omega_{2}
\end{aligned} \quad \alpha \delta-\beta \gamma=1
$$

where $\alpha, \beta, \gamma, \delta$ are integers. The curve as first expressed in terms of $p(u)$ and its derivatives is unaltered by ( $(8)$. In the new reference system the curve and each $\omega_{i j}$ are unaltered if $(8)$ is congruent to identity $(\bmod 7)$. On the other hand if $(8)$ is not congruent to identity $(\bmod 7)$, the $\omega_{i j}$ are permuted and we may look upon this operation either as merely a change in the coördinate system in which the curve is fixed or as a collineation in which the reference system is fixed and the $E^{7}$ passes into a new curve which belongs to $F$. Therefore all transformations $(8)$ which are congruent to identity $(\bmod 7)$ give rise to the identical collineation. These transformations constitute a subgroup of (8) of index $2 \cdot 168$. All elements of $(8)$ in a coset of this subgroup give rise to a collineation which permutes the curves of $F$ except the element $\left|\begin{array}{rr}-1 & 0 \\ 0 & -1\end{array}\right|$ which is the element $V$. Hence there are $2 \cdot 168 / 2$ or 168 collineations which interchange the members of $F$ * These collineations may be represented by the elements of (8) reduced modulo 7 , that is

$$
\begin{aligned}
& \omega_{1}^{\prime}=\alpha \omega_{1}+\beta \omega_{2} \\
& \omega_{2}^{\prime}=\gamma \omega_{1}+\delta \omega_{2}
\end{aligned} \quad \alpha \delta-\beta \gamma \equiv 1(\bmod 7)
$$

It is well known that any transformation of the group (8) is a combination of the transformations

$$
S: \omega^{\prime}=\omega+1 \quad T: \omega^{\prime}=-1 / \omega,
$$

where $S$ is of period 7 and $T$ is of period 2 when reduced modulo 7 . Since $T^{2} \equiv S^{7} \equiv(S T)^{3} \equiv\left(S^{4} T\right)^{4} \equiv 1 \dagger$, these relations define a $G_{168}$ of collineations on the reduced periods which permutes the members of the family $F$. Therefore we have the following theorem:

* See K. F., wol. 1, p. 398.

t It should be noted that in homogeneous form, $T$ is of period $4,\left(S^{4} T\right)$ is af period 8 . Hence $V$ and $T^{2}$ are the same. Compare Dickson, Linear Groups, p. 303. 
THEOREM I. The family $F$ of elliptic $E^{7}$ 's, each member of which is unaltered by $G_{2.7^{2}}$, is unaltered as a whole by a collineation group $G_{2 \cdot 7^{2 \cdot 168}}$ for which $G_{2 \cdot 7^{2}}$ is an invariant subgroup. Under the group of $F$ each curve belongs to a conjugate set of 168 curves. ${ }^{*}$

The collineation $T$ permutes the $H_{i}(i=\infty, 0,1, \ldots, 6)$ as follows: $(\infty 0)$, (16), (25), (34), where the subscripts only are used. The collineation $S$ permutes the $H_{i}(i=0,1, \ldots, 6)$ cyclically and leaves $H_{\infty}$ invariant. Under the group (8) the $H_{i}$ are permuted like the 8 points $\infty, 0,1, \ldots, 6$ in a finite geometry modulo 7 , there being 8 points on a line.

The equations of the collineations $S$ and $T$ in terms of $X_{i}$ are $\dagger$

$$
\begin{aligned}
& S: X_{i}^{\prime}=\epsilon^{i^{i} / 2} X_{i} \\
& T: X_{i}^{\prime}=c \sum_{\beta=0}^{6} \epsilon^{i \beta} X_{\beta} \quad(i=0,1, \ldots, 6) .
\end{aligned}
$$

5. The fixed spaces. In $G_{2 \cdot 7^{2}}$, the $7^{2}$ involutions $V_{i j}(i, j=0,1, \ldots, 6)$ have the form $u^{\prime}=-u+\omega_{i j}$. The fixed points of these involutions are $u \equiv \omega_{i j} / 2$ $+P / 2$ where $P / 2$ can evidently have the values $0, \omega_{1} / 2, \omega_{2} / 2$, and $\left(\omega_{1}+\omega_{2}\right) / 2$. We consider the simplest set, i. e., the set for which $i=j=0$.

Due to the involutory character of $V$, there are two skew spaces of fixed points in $S_{6}$, an $S_{2}$ and an $S_{3}$. If the coördinates of these fixed spaces be denoted by $y_{i}$ and $z_{j}(i=0,1,2,4 ; j=1,2,4)$ respectively, the equations of the transformation from the coördinates $X_{i}$ to those of $y$ and $z$ are

$$
\begin{array}{rlrl}
X_{0} & =y_{0}, & \\
X_{1}+X_{5} & =2 y_{1}, & X_{1}-X_{6}=2 z_{1}, \\
X_{2}+X_{5}=2 y_{2}, & X_{2}-X_{5}=2 z_{2}, \\
X_{4}+X_{3}=2 y_{4}, & X_{4}-X_{3}=2 z_{4} .
\end{array}
$$

In terms of $y$ and $z, V$ now has the form

$$
y_{i}^{\prime}=y_{i}, \quad z_{j}^{\prime}=-z_{j} \quad(i=0,1,2,4 ; j=1,2,4) .
$$

In (12), $y_{i}=0$ determine the $S_{2}$ of fixed points and $z_{j}=0$ determine the $S_{3}$ of fixed points. The fixed $S_{5}$ 's are either on $S_{2}$ with equations $\alpha_{0} y_{0}+\alpha_{1} y_{1}+\alpha_{2} y_{2}$ $+\alpha_{4} y_{4}=0$ or on $S_{3}$ with equations $\beta_{1} z_{1}+\beta_{2} z_{2}+\beta_{4} z_{4}=0$. The $\alpha$ 's may be determined by putting the $S_{5}$ on $u_{1}, u_{2}, u_{3}$, three arbitrary points on $E^{7}$, so that necessarily this $S_{5}$ cuts $E^{7}$ in the points $-u_{1},-u_{2},-u_{3}$. Therefore the $S_{5}$ contains the point $u=0$, but no proper half period point. Hence all the fixed $S_{5}$ 's on the $S_{2}$ and therefore $S_{2}$ itself, contain the point $u=0$ but no proper

* See K. F., vol. 1, p. 398.

† Compare K. F., vol. 2, p. 292. The formula for $S$ is compatible with Klein's for $n$ a prime number. As we deal with collineations in homogeneous forms we do not need to keep $c$ of the K. F. formula; it is therefore dropped in the remainder of the work. 
half period point. Therefore $S_{3}$ contains the proper half period points since . they are also fixed points.

The family $F$ of $E^{7}$ 's projected from the fixed $S_{2}$ upon the fixed $S_{3}$ becomes a family $F_{1}$ of rational cubics doubly covered, since the pairs $( \pm u)$ corresponding under $V$ each project into the same point. In a similar manner, by projection from $S_{3}$ upon $S_{2}, F$ becomes a family $F_{2}$ of conics doubly covered.

It is my purpose to discuss the families $F_{1}, F_{2}$, for which the curves in each family will vary with $\omega$ whereas the points on a particular curve will vary with the pairs $\left( \pm u\right.$ ) on the original $E^{7}$. The $G_{2 \cdot 7^{2} \cdot 168}$ has now reduced to a $G_{168}$ in $S_{2}$ and $S_{3}$ which leaves $F_{1}$ and $F_{2}$ invariant. This $G_{168}$ is generated by $S$ and $T$ whose equations are easily found to be

$$
\begin{aligned}
& S: \quad \begin{array}{l}
y_{i}^{\prime}=\epsilon^{-i^{2} / 2} y_{i} \\
z_{j}^{\prime}=\epsilon^{-j / 2} z_{j}
\end{array} \quad(i=0,1,2,4 ; j=1,2,4) \\
& T: \quad \begin{array}{l}
y_{i}^{\prime}=y_{0}+\sum_{j}\left(\epsilon^{i j}+\epsilon^{-i j}\right) y_{j} \quad(i=0,1,2,4) \\
z_{k}^{\prime}=\sum_{l}\left(\epsilon^{l k}-\epsilon^{-l k}\right) z_{l} \quad(j, k, l=1,2,4) .
\end{array}
\end{aligned}
$$

Formulas (12), (13) and (14) constitute a second canonical system of coördinates for $E^{7}$.

\section{THE QUADRICS ON $E^{7}$}

1. The pencil of quadrics on $E^{7}$. Hermite has shown that the number of linearly independent quadrics on $E^{7}$ is fourteen. These fourteen quadrics cut out the $E^{7}$ completely with no extraneous intersection.* In the second system of coördinates a general quadric has the form

$$
q_{\circ}=\sum_{i, k=0}^{6} \alpha_{i k} X_{i} X_{k}=0,
$$

where $\alpha_{i k}$ are constants. Let us suppose that the $\alpha_{i k}$ are so determined that the quadric contains the curve $E^{7}$. Under the collineation $S_{10}, E^{7}$ is transformed into itself. Hence the quadric (15) is transformed into a quadric on. $E^{7}$. The transforms of $q_{0}$ under $S_{10}$ are of the form

$$
q_{j}=\sum_{i, k=0}^{6} \alpha_{i k} \epsilon^{j(i+k)} X_{i} X_{k}=0 \quad(j=0,1, \ldots, 6) .
$$

Since each $q_{j}$ is on $E^{7}$, a linear combination of them will be on the curve. Multiplying each $q_{j}$ by unity and adding we obtain a particular quadric $Q_{0}$ on $E^{7}$ characterized by the fact that it consists only of those terms for which $i+k \equiv 0$ $(\bmod 7)$. Using the multipliers $1, \epsilon^{5}, \epsilon^{3}, \epsilon^{1}, \epsilon^{6}, \epsilon^{4}, \epsilon^{2}$, respectively, we obtain a

\footnotetext{
* Compare K. F., vol. 2, p. 245.
} 
second particular quadric $Q_{1}$ on $E^{7}$ characterized by the fact that it consists only of those terms for which $i+k \equiv 1(\bmod 7)$. Proceeding in this way we obtain 7 particular quadrics on $E^{7}$. They are

$$
\begin{aligned}
Q_{i}=\alpha_{i 0} X_{i}^{2}+2 \alpha_{i 1} X_{i+1} X_{i-1}+2 \alpha_{i 2} X_{i+2} X_{i-2}+2 \alpha_{i 4} X_{i+4} X_{i 4} \\
=0(i=0,1, \ldots, 6) .
\end{aligned}
$$

Any quadric on the curve $E^{7}$ is a linear combination of the $Q^{\prime}$ s, since the seven $Q$ 's contain as yet 28 arbitrary coefficients. But since each $Q_{i}$ is sent into $Q_{i+1}$ by $S_{01}$, these 28 coefficients reduce to four, i. e., $\alpha_{0}, \alpha_{1}, \alpha_{2}, \alpha_{4}$. From these seven $Q$ 's, we know that we must be able to get the 14 linearly independent quadrics on the $E^{7}$. The $\alpha^{\prime}$ 's therefore must contain a parameter linearly and there will be one quadric of the type $Q_{i}$ for which a particular $\alpha$ will vanish. ${ }^{*}$ At most, then, a pencil can arise from the four terms of each $Q_{i}$. Any one of these seven pencils is defined by the fact that it admits one of the seven dihedral $G_{2.7}$ 's whose cyclic subgroup is $S_{10}$. For example $Q_{0}$ admits the dihedral $\left(S_{10} V\right)$.

Since the $\alpha$ 's contain a parameter linearly, they may be interpreted as the coördinates of a point on a line in an $S_{3}$. By choosing two members from the pencil of quadrics, the line is determined. We shall determine the $\alpha$ 's later as functions of $\omega$ and the parameter just mentioned.

2. The group on the quadrics. Under $G_{2 \cdot 7^{2}}$ each member of the family $F$ of $E^{7} \mathrm{~s}$ is transformed into itself and the quadrics on each curve are transformed into quadrics on that curve, so that a group of collineations is induced upon the $Q_{i}$ as variables. Moreover since $S$ and $T$ interchange the members of the 168 sets of conjugate curves, they will send the quadrics on a given curve into a linear combination of the quadrics on the transformed curve. If we indicate the group $G_{2 \cdot 7 \cdot 168}$ on the $X_{i}$ 's in (7) and (11) by $G(\epsilon)$, then the induced group on the quadrics $Q_{i}$ is $G\left(\epsilon^{2}\right)$.

In order to express all the quadrics (17) by one equation, consider the general quadric obtained by taking a linear combination of them. Such a quadric has the form.

$$
\sum_{i=0}^{6} L_{i} Q_{i}=0
$$

where the $L_{i}$ are arbitrary constants. On a given curve of $F$ determined by a proper set of values of $\alpha_{i}(i=0,1,2,4)$, the bilinear form (18) is an ilentity in $L$ and $u$. If we require that this bilinear form be an invariant under $G\left(\epsilon^{2}\right)$, there will be a certain group induced upon the $L_{i}$ as variables. This group on the variables $L_{i}$ is $G\left(\epsilon^{-2}\right)$.

\footnotetext{
* Compare K. F., vol. 2, p. 268. Klein obtained the quadrics on the elliptic curves from the three-term sigma relation.
} 
3. A Kleinian form. Since the properties of the groups on the $L_{i}$ and $Q_{i}$ are the same as those on the $X_{i}$, we isolate one of the involutions in the $L_{i}, Q_{i}$ groups, i. e., that one induced by $V$ which was isolated in the $X_{i}$ group. We introduce the variables $v$ and $u, \zeta$ and $\vartheta$ with $Q_{z}$ and $L_{i}$, respectively, as $y$ and $z$ were introduced with the $X_{i}$. The equations of the transformations from $Q_{i}$ and $L_{i}$ to $v, u, \zeta$ and $\vartheta$ can be written down as were those for $y$ and $z$. After this change of variables, (18) has the form

$$
\begin{aligned}
F^{\prime} & =\zeta_{0}\left[\alpha_{0} y_{0}^{2}+2 \alpha_{1} y_{1}^{2}+2 \alpha_{2} y_{2}^{2}+2 \alpha_{4} y_{4}^{2}-2 \alpha_{1} z_{1}^{2}-2 \alpha_{2} z_{2}^{2}-2 \alpha_{4} z_{4}^{2}\right] \\
& +2 \zeta_{1}\left[\alpha_{0} y_{1}^{2}+2 \alpha_{1} y_{0} y_{2}+2 \alpha_{2} y_{1} y_{4}+2 \alpha_{4} y_{2} y_{4}+\alpha_{0} z_{1}^{2}+2 \alpha_{2} z_{1} z_{4}-2 \alpha_{4} z_{2} z_{4}\right] \\
& +2 \zeta_{2}\left[\alpha_{0} y_{2}^{2}+2 \alpha_{1} y_{1} y_{4}+2 \alpha_{2} y_{0} y_{4}+2 \alpha_{4} y_{1} y_{2}-2 \alpha_{1} z_{1} z_{4}+\alpha_{0} z_{2}^{2}+2 \alpha_{4} z_{1} z_{2}\right] \\
& +2 \zeta_{4}\left[\alpha_{0} y_{4}^{2}+2 \alpha_{1} y_{2} y_{4}+2 \alpha_{2} y_{1} y_{2}+2 \alpha_{4} y_{0} y_{1}+2 \alpha_{1} z_{2} z_{4}-2 \alpha_{2} z_{1} z_{2}+\alpha_{0} z_{4}^{2}\right] \\
& +4 \vartheta_{1}\left[\alpha_{0} y_{1} z_{1}+\alpha_{1} y_{0} z_{2}-\alpha_{2}\left(y_{1} z_{4}+y_{4} z_{1}\right)+\alpha_{4}\left(y_{2} z_{4}-y_{4} z_{2}\right)\right] \\
& +4 \vartheta_{2}\left[\alpha_{0} y_{2} z_{2}+\alpha_{1}\left(y_{4} z_{1}-y_{1} z_{4}\right)+\alpha_{2} y_{0} z_{4}-\alpha_{4}\left(y_{1} z_{2}+y_{2} z_{1}\right)\right] \\
& +4 \vartheta_{4}\left[\alpha_{0} y_{4} z_{4}-\alpha_{1}\left(y_{2} z_{4}+y_{4} z_{2}\right)+\alpha_{2}\left(y_{1} z_{2}-y_{2} z_{1}\right)+\alpha_{4} y_{0} z_{1}\right]=0 .
\end{aligned}
$$

On $E^{\gamma}$ the above form is an identity in $\zeta, \vartheta$ and can be separated into seven parts. However we shall have occasion to separate it into two parts, $P_{1}$ and $P_{2}$, such that the part $P_{1}$ contains the coefficients $\zeta$ and the part $P_{2}$ the coefficients $\vartheta$. The part $P_{1}$ is partly symmetrical and partly alternating in the coefficients $\alpha$ and $\zeta$, hence the $\zeta$ 's can be interpreted as the coördinates of a point on a line in an $S_{3}$ and are therefore cogredient to the $\alpha$ 's. Hence we may conclude this section with the theorem

THEOREM II. $F^{\prime}$ is a Kleinian form which remains invariant under the simultaneous transformation by the isomorphic groups $M(\epsilon)$ of (14) on the variables $y$ and $z ; M\left(\epsilon^{-2}\right)$ on the variables $\zeta$ and $\alpha$ and $\vartheta$. The form $F^{\prime}$ determines the curve $E^{7}$ uniquely when the modular functions $\alpha$ are properly given, $i . e$. subject to the relation which connects their ratios.

\section{THE INTERPRETATION OF THE FORM $F^{\prime}$}

1. Its fundamental elliptic mcdular functions. Each curve of the family $F$ has on it the point whose parameter is $u=0$. As $\omega=\omega_{1} / \omega_{2}$ varies this zero point generates a locus. It has already been pointed out that the zero point is in the space $S_{2}$ of fixed points, i. e., when $u=0$ all the $y$ 's vanish. Let $z_{\imath}=t_{i}(i=$ $1,2,4)$ for $u=0$; then $F^{\prime}$ in (19) reduces to

$$
\begin{aligned}
\zeta_{0}[0 & \left.-2 \alpha_{1} t_{1}^{2}-2 \alpha_{2} t_{2}^{2}-2 \alpha_{4} t_{4}^{2}\right] \\
& +2 \zeta_{1}\left[\alpha_{0} t_{1}^{2}-0+2 \alpha_{2} t_{1} t_{4}-2 \alpha_{4} t_{2} t_{4}\right] \\
& +2 \zeta_{2}\left[\alpha_{0} t_{2}^{2}-2 \alpha_{1} t_{1} t_{4}-0+2 \alpha_{4} t_{1} t_{2}\right] \\
& +2 \zeta_{4}\left[\alpha_{0} t_{4}^{2}+2 \alpha_{1} t_{2} t_{4}-2 \alpha_{2} t_{1} t_{2}-0\right] \equiv 0 .
\end{aligned}
$$


Since (20) is an identity in the $\zeta_{i}$, their coefficients must vanish. These coefficients are linear in the $\alpha$ 's, all of which do not vanish simultaneously, therefore the determinant of the $\alpha$ 's must vanish. After removing numerical factors, we find a skew-symmetric determinant of even order. This determinant is a perfect square. ${ }^{*}$ It furnishes in variables $t_{i}$ Klein's quartic, which is denoted as follows:

$$
K=t_{1}^{3} t_{2}+t_{2}^{3} t_{4}+t_{4}^{3} t_{1}=0
$$

$K$ is the equation of the locus of the zero point of the family of $E^{7} \mathrm{~s}$ and admits a group $G_{168}$ of collineations into itself, cogredient to the group in $z$ in (14). The ratios $t_{1}: t_{2}: t_{4}$ are the fundamental elliptic modular functions of the form $F^{\prime}$. The expressions for these ratios as uniform functions of the modulus $\omega$ may be obtained by setting $u=0$ in the expressions for the $z$ 's in terms of $u, \omega_{1}, \omega_{2}$, as indicated.

Since the curve $E^{7}$ varies with $\omega$, and since each $E^{7}$ possesses a zero point, i. e., a point $t$ which is on $K$, it is clear that the variation of $E^{7}$ with $\omega$ may be imaged by the variation of $t$ on $K$. We shall express other elliptic modular functions associated with the family of $E^{7}$ 's in terms of the $t_{i}$.

2. The null-system. The form in (20) is a null-system, since it can be written in the form

$$
\left(\alpha_{0} \zeta_{1}\right) t_{1}^{2}+\left(\alpha_{0} \zeta_{2}\right) t_{2}^{2}+\left(\alpha_{0} \zeta_{4}\right) t_{4}^{2}+2\left(\alpha_{4} \zeta_{2}\right) t_{1} t_{2}+2\left(\alpha_{1} \zeta_{4}\right) t_{2} t_{4}+2\left(\alpha_{2} \zeta_{1}\right) t_{4} t_{1}=0
$$

where $\left(\alpha_{i} \zeta_{k}\right)=\alpha_{i} \zeta_{k}-\alpha_{k} \zeta_{i}$. Since (20) vanishes independently of the $\zeta$ 's it represents a singular null-system. $\dagger$ Hence (22) is the equation of a line whose coördinates may be taken as

$$
\begin{array}{ll}
\left(\alpha_{0} \zeta_{1}\right)=+2 t_{1} t_{2}, & \left(\alpha_{4} \zeta_{2}\right)=t_{1}^{2} \\
\left(\alpha_{0} \zeta_{2}\right)=+2 t_{2} t_{4}, & \left(\alpha_{1} \zeta_{4}\right)=t_{2}^{2} \\
\left(\alpha_{0} \zeta_{4}\right)=+2 t_{4} t_{1}, & \left(\alpha_{2} \zeta_{1}\right)=t_{4}^{2},
\end{array}
$$

where $\alpha$ is clearly a point on a line. Since the coördinates of the line of the $\alpha$ 's are functions of $t$, we shall call it the modular line and denote it by $L_{m}$. The intersection of the coördinate planes of the reference tetrahedron in the space of the $\alpha$ 's, an $S_{3}^{(\alpha)}$, with $L_{m}$ furnishes four convenient sets of values of the $\alpha$ 's, which substituted in $F^{\prime}$ give rise to the 28 quadrics on $E^{7}$, of which only 14 are linearly independent, since any two sets of the $\alpha$ 's are linear combinations of the remaining two sets. These sets of values are

* Burnside and Panton, Theory of Equations, vol. 2, p. 46.

† See Veblen and Young, Projective Geometry, vol. 1, p. 324. 


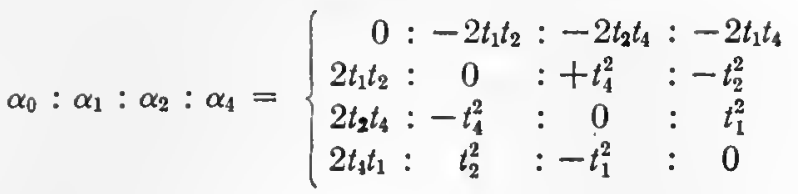

The sets (24) suggest that we make a transformation on the $\alpha$ 's in $F^{\prime}$. Let $\xi$ be a plane such that it intersects $L_{m}$ in the point $\alpha$. From (24) we find this transformation to be

$$
\begin{aligned}
& \alpha_{0}=0+2 t_{1} t_{2} \xi_{1}+2 t_{2} t_{4} \xi_{2}+2 t_{4} t_{1} \xi_{4} \\
& \alpha_{1}=-2 t_{1} t_{2} \xi_{0}+0-t_{4}^{2} \xi_{2}+t_{2}^{2} \xi_{4} \\
& \alpha_{2}=-2 t_{2} t_{4} \xi_{0}+t_{4}^{2} \xi_{1}+0 \quad-t_{1}^{2} \xi_{4} \\
& \alpha_{4}=-2 t_{4} t_{1} \xi_{0}-t_{2}^{2} \xi_{1}+t_{1}^{2} \xi_{2}+0 .
\end{aligned}
$$

If $F^{\prime}$ is transformed by (25), it will take the form

$$
\sum \xi_{i} \zeta_{j} \phi_{i j}+\sum \xi_{i} \vartheta_{l} \phi_{i l}^{\prime}=0 \quad(i, j=0,1,2,4 ; l=1,2,4) .
$$

The 28 quadrics on the curve $E^{7}$, of which naturally only 14 are linearly independent, are found by equating to zero the coefficients of the terms $\xi_{i} \zeta_{j}$ and $\xi_{i} \vartheta_{l}$ respectively, i. e., the $\phi_{i j}$ and $\phi_{i l}$. We shall have occasion to use all of these quadrics, but will refer to them briefly in the above notation.

3. The rational curves in $S_{2}$ and $S_{3}$. We have seen that $\alpha$ and $\zeta$ are cogredient variables and that $P_{1}$ is partly alternating and partly symmetrical in $\alpha$ and $\zeta$. We now rewrite $P_{1}$ so as to exhibit this property. It has the form

$$
\begin{gathered}
\alpha_{0} \zeta_{0} y_{0}^{2}+4 \sum \alpha_{1} \zeta_{1} y_{0} y_{2}+2 \sum\left(\alpha_{0} \zeta_{1}+\alpha_{1} \zeta_{0}\right) y_{1}^{2}+4 \sum\left(\alpha_{2} \zeta_{4}+\alpha_{4} \zeta_{2}\right) y_{1} y_{2} \\
+2 \sum\left(\alpha_{0} \zeta_{1}\right) z_{1}^{2}+4 \sum\left(\alpha_{4} \zeta_{2}\right) z_{1} z_{2}=0
\end{gathered}
$$

where $\Sigma$, unless otherwise denoted, refers to the cyclic advance of the subscripts $1,2,4$. This form furnishes the means by which the projections of the family $F$ of $E^{7}$ 's upon the fixed spaces $S_{2}$ and $S_{3}$ are found. The second part $P_{2}$, bilinear in $y$ and $z$, does not enter in these projections, since it vanishes when either space is considered separately.

Since $\zeta$ is perfectly arbitrary, consider it on the modular line $L_{m}$. Now interchange $\alpha$ and $\zeta$ in (27). The new form is similar to the old except that the sign of each term in $z$ is changed. Denote the transformed $P_{1}$ by $P_{1}^{\prime}$. Since $P_{1}$ in (27) is a quadric on $E^{7}$ and since we consider $\zeta$ on $L_{m}, P_{1}^{\prime}$ is also a quadric on $E^{7}$. Whence their sum $P_{1}+P_{1}^{\prime}$ and their difference $P_{1}-P_{1}^{\prime}$ are quadrics on $E^{7}$. Consider the former;

$$
\alpha_{0} \zeta_{0} y_{0}^{2}+4 \sum \alpha_{1} \zeta_{1} y_{0} y_{2}+2 \sum\left(\alpha_{0} \zeta_{1}+\alpha_{1} \zeta_{0}\right) y_{1}^{2}+4 \sum\left(\alpha_{2} \zeta_{4}+\alpha_{4} \zeta_{2}\right) y_{1} y_{2}=0
$$


The equation (28) for arbitrary $\alpha$ and $\zeta$ on $L_{m}$ furnishes a system of quadrics in $S_{3}$ which intersect in a cubic curve. From the symmetry of $\alpha$ and $\zeta$ in (28), we lose no generality by setting $\alpha_{i}=\zeta_{i}$. We then have

$$
\alpha_{0}^{2} y_{0}^{2}+4 \sum \alpha_{1}^{2} y_{0} y_{2}+4 \sum \alpha_{0} \alpha_{1} y_{1}^{2}+8 \sum \alpha_{2} \alpha_{4} y_{1} y_{2}=0 .
$$

Since $\alpha$ is linear in a parameter $\lambda$ on $L_{m}$, (29) furnishes a system of quadrics quadratic in $\lambda$. The coefficients of this quadratic system of quadrics are functions of $t$, so that as $t$ varies on $K$, we get a family $F_{1}$ of cubic curves $C^{3}$ in $S_{3}$. Hence we may state the following theorem:

(30) THEOREM III. The projection $C^{3}$ of the curve $E^{7}$ upon $S_{3}$ is the base curve of the quadratic system of quadrics (29).

Consider now the difference $P_{1}-P_{1}^{\prime}$. This is a conic in $S_{2}$. It has the form

$$
\sum\left(\alpha_{0} \zeta_{1}\right) z_{1}^{2}+2 \sum\left(\alpha_{4} \zeta_{2}\right) z_{1} z_{2}=0
$$

which from (23) may be written as follows:

$$
\sum t_{1} t_{2} z_{1}^{2}+\sum t_{1}^{2} z_{1} z_{2}=0
$$

This shows that the system of conics varies with $t$ on $K$. It is the polar conic of $K$ as to $z$. Hence the theorem:

(33) THEOREM IV. The projection $C^{2}$ of the family $F$ of $E^{7} s$ upon $S_{2}$ is the system of polar conics of Klein's quartic $K$.

4. The net of quadrics in $S_{3}(\alpha)$. The quadric in (29) will be the square of a plane when the rank of its discriminant is 1 . Its discriminant is of rank 1 if only the three relations

$$
\alpha_{0} \alpha_{2}-\alpha_{4}^{2}=0, \quad \alpha_{0} \alpha_{4}-\alpha_{1}^{2}=0, \quad \alpha_{0} \alpha_{1}-\alpha_{2}^{2}=0,
$$

are satisfied.

Consider now the net of quadrics

$$
t_{1}\left(\alpha_{0} \alpha_{2}-\alpha_{4}^{2}\right)+t_{2}\left(\alpha_{0} \alpha_{4}-\alpha_{1}^{2}\right)+t_{4}\left(\alpha_{0} \alpha_{1}-\alpha_{9}^{2}\right)=0
$$

From the transformations $S$ and $T$ in (14) we conclude that (35) is a Kleinian form.

The discriminant of the net (35) is $K$. Hence so long as $t$ is on $K$, the quadric (35) has a double point. If we border the discriminant (35) with variables $\xi$ and expand, we find the equation of this double point to be

$$
(\alpha \xi)^{2}=\xi_{0}^{2} 4 t_{1} t_{2} t_{4}+\sum\left(-t_{1}^{3}-t_{2}^{2} t_{4}\right) \xi_{1}^{2}+\sum\left(2_{1} t_{4}^{2}\right)\left(2 \xi_{0} \xi_{1}\right)+\sum\left(2 t_{1}^{2} t_{4}\right)\left(2 \xi_{1} \xi_{2}\right)=0
$$


Therefore the coördinates of the double-point are

$$
\begin{aligned}
& \rho \alpha_{0}=\sqrt{4 t_{1} t_{2} t_{4}} \quad: 2 t_{1} t_{2} t_{4}: \quad t_{1} t_{4}^{2} \quad: \quad t_{1}^{2} t_{2}:: \quad t_{2}^{2} t_{4} \\
& \rho \alpha_{1}=\sqrt{-t_{1}^{3}-t_{2}^{2} t_{4}}: \quad t_{1} t_{4}^{2}:-t_{1}^{3}-t_{2}^{2} t_{4}: \quad t_{1}^{2} t_{4} \quad: \quad t_{4}^{2} t_{2} \\
& \rho \alpha_{2}=\sqrt{-t_{2}^{3}-t_{4}^{2} t_{1}}: \quad t_{2} t_{1}^{2}: \quad t_{1}^{2} t_{4} \quad:-t_{2}^{3}-t_{4}^{2} t_{1}: \quad t_{2}^{2} t_{1} \\
& \rho \alpha_{4}=\sqrt{-t_{4}^{3}-t_{1}^{2} t_{2}}: \quad t_{4} t_{2}^{2}: \quad t_{4}^{2} t_{2} \quad: \quad t_{2}^{2} t_{1} \quad:-t_{4}^{3}-t_{1}^{2} t_{2}
\end{aligned}
$$

where $\rho$ is $1, \alpha_{0}, \alpha_{1}, \alpha_{2}, \alpha_{4}$, respectively. That is to say, we can express the entire system (37) rationally and without extraneous factors by giving the ten quadratic combinations of the $\alpha$ 's. These combinations are the coefficients of the terms $\xi_{i} \xi_{k}$ in (36).

The order of the linear modular group in the space of the $y$ 's and $\alpha$ 's is double the order of the group* in the space of the $z$ 's, that is, the group is a $G_{2 \cdot 168}$, due to the fact that the identical collineation appears in the form $y_{i}^{\prime}= \pm y_{i}$. Hence the coördinates of a modular-point or plane in $S_{3}{ }^{(\alpha)}$ and likewise in $S_{3}$ cannot be expressed rationally in terms of the $t_{t}$ without an extraneous factor. The coördinates may however be expressed irrationally in terms of $t$ as above, and it is to be noted that their ratios are uniform functions of $\omega$.

A number of such modular root functions are suggested by the geometry of the system of cubic curves $C^{3}$ in $S_{3}$. Thus the locus of the zero point on the curves $C^{3}$, the locus of the plane of the half period points, the locus of the point where the tangent at the zero point meets the half period plane, as well as the transforms of these points and planes in the null-system of $C^{3}$, give rise to functions of this type. Some of these are determined later.

The locus of the double point (36) as $t$ varies on $K$ is a well known space curve $J$ of order 6 in $S_{3}{ }^{(\alpha)}, \dagger$ whose points are in a one-to-one correspondence with the points of $K$. If we border the discriminant of (35) with $\xi$ and $\eta$, which are to be thought of as parameters, we have $\infty{ }^{6}$ curves of the third order in $t$ which intersect $K$ in 12 points which correspond to the 12 meets of the planes $\xi, \eta$ with $J$. Hence when $\xi=\eta$ the cubic in $t$ will be a contact cubic of $K$. Thus the system (36) for variable $\xi$ is a system of contact curves of the third order associated with $J . \ddagger$

5. The modular line and spread. If a point $y$ be taken on $J$, a quadric of the net (35) has a node at $y$ and the polar plane of this point as to this quadric vanishes, while the polar planes of the other two quadrics meet in a line. Take the coördinates of the point $y$ on $J$ as those in the second column of (37). The three

* Compare K. F., vol. 2, p. 313.

† Compare Snyder and Sisam, Analytic Geometry of Space, p. 168.

$\ddagger$ See K. F., vol. 1, p. 716 . 
polar planes of this point as to the quadrics in the net (35) are in a pencil, and have the form

$$
\begin{aligned}
& \alpha_{0} t_{1}^{2}-0+2 \alpha_{2} t_{1} t_{4}-2 \alpha_{4} t_{2} t_{4}=0 \\
& \alpha_{0} t_{2}^{2}-2 \alpha_{1} t_{1} t_{4}-0+2 \alpha_{4} t_{1} t_{2}=0 \\
& \alpha_{0} t_{4}^{2}+2 \alpha_{1} t_{2} t_{4}-2 \alpha_{2} t_{1} t_{2}-0=0 .
\end{aligned}
$$

The axis of the pencil of planes (38) is the modular line $L_{m}$. Every point on $L_{m}$ is in a one-to-one correspondence with the point $y$ on $J$. Since the coördinates of $L_{m}$ and of the point $y$ on $J$ are functions of $t$, the variation of $y$ and of $L_{m}$ also may be imaged by the variation of $t$ on $K$. Hence as $y$ generates $J$, $L_{m}$ generates a ruled surface of order 8 . That $M$ is of order 8 may be shown as follows. The condition that a line $l$ meet $L_{m}$ is a linear condition on their coördinates, or a conic in $t$. This conic in $t$ meets $K$ in 8 points to each of which there corresponds a meet of $l$ and $M$, whence $M$ is of order 8 .

Let us now consider the general quadric $Q$ in the net (35), and put on it the condition that it have a node. The four partial derivatives $\partial Q / \partial \alpha_{i}$ must then vanish simultaneously. These are

$$
\begin{aligned}
0+\alpha_{1} t_{4}+\alpha_{2} t_{1}+\alpha_{4} t_{2} & =0, \\
\alpha_{0} t_{4}-2 \alpha_{1} t_{2} & =0, \\
\alpha_{0} t_{1}-2 \alpha_{2} t_{4} & =0, \\
\alpha_{0} t_{2}-2 \alpha_{4} t_{1} & =0 .
\end{aligned}
$$

The discriminant of these equations is $K$. If we eliminate $t$ from the equations (39), we find four cubic surfaces on each of which is $J$. Hence their common intersection is $J$. The equations of these are obtained from the vanishing of the third order determinants in the matrix of the equations (39). They are

$$
\begin{aligned}
& S_{1}=\alpha_{0}^{3}-8 \alpha_{1} \alpha_{2} \alpha_{4}=0, \\
& S_{2}=\alpha_{0}^{2} \alpha_{4}+2 \alpha_{0} \alpha_{2}^{2}+4 \alpha_{1} \alpha_{2}^{2}=0, \\
& S_{3}=\alpha_{0}^{2} \alpha_{1}+2 \alpha_{0} \alpha_{4}^{2}+4 \alpha_{2} \alpha_{4}^{2}=0, \\
& S_{4}=\alpha_{0}^{2} \alpha_{2}+2 \alpha_{0} \alpha_{1}^{2}+4 \alpha_{4} \alpha_{1}^{2}=0 .
\end{aligned}
$$

The modular spread $M$ multiplied by $\alpha_{0}$ is the following combination of $S$ in (40):

$$
S_{1}^{3}-8 S_{2} S_{3} S_{4}=\alpha_{0} M=0 .
$$

From this result it is evident that $J$ is a triple curve on $M$. Further, it can be shown that through every point of $J$ there pass three trisecants of $J$ and that $L_{m}$ itself is a trisecant of $J .^{*}$

This section can be partially summarized in the following theorem:

* The equation of $M$ and the facts concerning $J$ are easily obtained from a Cremona transformation of the third order. 
THEOREM V. Through every point $\alpha\left(=\alpha_{0}, \alpha_{1}, \alpha_{2}, \alpha_{4}\right)$ on the octavic ruled surface $M$ there passes a line $L_{m}$ and the pencil of points $\alpha$ on $L_{m}$ set in the form $F^{\prime}$ determines the quadrics on the curve $E^{7}$. As the line $L_{m}$ varies on $M$, the $E^{7}$ varies in the family $F$. The line $L_{m}$ (itself a trisecant of $J$ ) meets the triple curve $J$ on $M$ in three points which correspond to the three trisecants of $J$ that meet in a point $t$ of $J$. Thus the points $t$ of $J$ are in a one-to-one correspondence with the curves of the family of $E^{\prime \prime}$ s.

This completes the determination of the coefficients $\alpha$ of the quadrics $F^{\prime}$ which define the curve $E^{7}$.

\section{THE LOCI IN $S_{3}$}

1. The net of quadrics in $S_{3}$. In (35) a net of quadrics in $S_{3}{ }^{(\alpha)}$ was considered. The modular line $I_{m}$ and the modular spread were associated with this net. Consider now a similar net of quadrics in plane coördinates $U$ in $S_{3}$, and let us find the condition that this net have a double plane. From the contragredient transformations $S$ and $T$ on the $y^{\prime}$ ' in $(14)^{*}$, we conclude that the following net is a Kleinian form:

$$
t_{1}\left(2 U_{0} U_{1}-U_{2}^{2}\right)+t_{2}\left(2 U_{0} U_{2}-U_{4}^{2}\right)+t_{4}\left(2 U_{0} U_{4}-U_{1}^{2}\right)=0 .
$$

The discriminant of this net is $K$. The bordered form of the discriminant is the square of a plane in point coördinates, i. e.,

$$
t_{1} t_{2} t_{4} y_{0}^{2}+\sum\left(-t_{2}^{3}-t_{1} t_{4}^{2}\right) y_{1}^{2}+2 \sum t_{1}^{2} t_{2} y_{0} y_{1}+2 \sum t_{1} t_{2}^{2} y_{1} y_{2}=0 .
$$

So long as $t$ is on $K$, the coördinates of the double plane (43) are

$$
\begin{aligned}
& \rho U_{0}=\sqrt{t_{1} t_{2} t_{4}} \quad: t_{1} t_{2} t_{4}: t_{1}^{2} t_{2}: t_{2}^{2} t_{4}:: t_{4}^{2} t_{1}, \\
& \rho U_{1}=\sqrt{-t_{2}^{3}-t_{1} t_{4}^{2}}: t_{1}^{2} t_{2}:-t_{2}^{3}-t_{1} t_{4}^{2}: \quad t_{1} t_{2}^{2}: t_{1}^{2} t_{4} \text {, } \\
& \rho U_{2}=\sqrt{-t_{4}^{3}-t_{2} t_{1}^{2}}: \quad t_{2}^{2} t_{4}: \quad t_{1} t_{2}^{2}:-t_{4}^{3}-t_{2} t_{1}^{2}: \quad t_{2} t_{4}^{2} \text {, } \\
& \rho U_{4}=\sqrt{-t_{1}^{3}-t_{4} t_{2}^{2}}: \quad t_{4}^{2} t_{1}: \quad t_{1}^{2} t_{4} \quad: \quad t_{2} t_{4}^{2} \quad:-t_{1}^{3}-t_{4} t_{2}^{2} \text {, }
\end{aligned}
$$

where $\rho$ is $1, U_{0}, U_{1}, U_{2}, U_{4}$, respectively. As in (37), we may express the entire system in (44) by taking the 10 quadratic combinations of the $U$ 's from (43). The remarks following (37) apply here. The plane coördinates $U_{i}$ taken from the second column of (44) are the modular systems $A_{\gamma}$ developed by Klein. $\dagger$

With the net (42) there will be a modular line $L_{m}^{\prime}$, four cubic surfaces $S_{i}^{\prime}$, a modular surface $M^{\prime}$ and a sextic $J^{\prime}$. The coördinates of $L_{m}^{\prime}$ can be developed

* See K. F., vol. 1, p. 719.

† See K. F., vol. 1, p. 719. 
in a manner similar to that used in finding those of $L_{m}$ as the axis of the pencil of planes (38). They are

$$
\begin{array}{ll}
\left(U_{0} U_{1}^{\prime}\right)=t_{1} t_{2}, & \left(U_{2} U_{1}^{\prime}\right)=t_{1}^{2}, \\
\left(U_{0} U_{2}^{\prime}\right)=t_{2} t_{4}, & \left(U_{4} U_{2}^{\prime}\right)=t_{2}^{2}, \\
\left(U_{0} U_{4}^{\prime}\right)=t_{4} t_{1}, & \left(U_{1} U_{4}^{\prime}\right)=t_{4}^{2} .
\end{array}
$$

To every position of the plane $(U y)=0$ in (43) we have a line $L_{m}^{\prime}$ whose coördinates are given in (45). Since the coefficients of the plane $(U y)=0$ and $L_{m}^{\prime}$ are functions of $t$, the variation of the plane $(U y)$ and $L_{m}^{\prime}$ also may be imaged as the variation of $t$ on $K$. It should be noted that the space of the $\alpha$ 's is different from the space of the $y^{\prime}$ s. Hence the modular lines $L_{m}, L_{m}^{\prime}$; the curves $J, J^{\prime}$; the spreads $M, M^{\prime}$; and the cubic surfaces $S_{i}, S_{i}^{\prime}$ are all distinct.

2. The plane of the half period points. For the three half period points, the $z$ 's all vanish. If in the 14 linearly independent quadrics on $E^{7}$ we set the $z^{\prime}$ s all zero, we then obtain 8 quadrics in $y$ (since 6 of the 14 quadrics are bilinear in $y$ and $z$ and vanish for $z$ set equal zero). These 8 quadrics must pass through the half period points. If we call the plane of these points $(U y)$, then we should be able to obtain from these $S$ quadrics the four combinations $y_{i}(U y)(i=0$, $1,2,4)$. The combinations furnishing these types of quadrics come from the systems

$$
\begin{gathered}
t_{1} t_{4}^{3} \phi_{12}+t_{1} t_{2}^{2} t_{4} \phi_{14}-t_{1}^{2} t_{2}^{2} \phi_{24}-t_{2}^{2} t_{4}^{2} \phi_{21}+t_{2} t_{4}^{3} \phi_{40}=0 \\
t_{1} \phi_{41}+t_{2} \phi_{01}-t_{4} \phi_{04}=0 \\
t_{2} \phi_{12}+t_{4} \phi_{02}-t_{1} \phi_{01}=0 \\
t_{4} \phi_{24}+t_{1} \phi_{04}-t_{2} \phi_{02}=0
\end{gathered}
$$

The common factor $(U y)$ obtained from these equations (46) when the $z$ 's are zero is precisely the plane

$$
(U y)=t_{1} t_{2} t_{4} t y_{0}+t_{1}^{2} t_{2} y_{1}+t_{2}^{2} t_{4} y_{2}+t_{4}^{2} t_{1} y_{4}=0,
$$

whose square appeared in (43). Hence the coordinates of the half period plane are the modular functions set forth in (44).

Since the half period plane is of the form $\Sigma \alpha_{i} y_{i}=0(i=0,1,2,4)$, and since it may be considered as an $S_{5}$ in $S_{6}$, it contains the point $u=0$ and three pairs of points $( \pm \imath)$ on $E^{7}$, since the three pairs are sufficient to determine the $\alpha$ 's. It is therefore a fixed $S_{5}$ on the fixed $S_{2}$. Since the pairs $( \pm u)$ are the half period points, they are coincident points in $S_{6}$, hence the half period plane (47) considered as an $S_{5}$ is a tritangent hyperplane of $E^{7}$, tangent at the points $\omega_{1} / 2, \omega_{2} / 2$ and $\left(\omega_{1}+\omega_{2}\right) / 2$ and passing through the point $u=0$.

* We draw from the entire system of quadrics $\phi_{i j}(16$ in number) for convenience. These $\phi_{i j}$ are the coefficients of the terms $\xi_{i} \xi_{i}$ in $F^{\prime}$ after the transformation in (25). 
Let us now consider the systems of quadrics in (46) with the $z$ 's different from zero. These expressed in terms of $y$ and $z$ are

$$
\begin{aligned}
& t_{4} y_{0}(U y)=+2 t_{1} t_{2}^{2} z_{2}(41)+\left(-2 t_{1} t_{2} t_{4} z_{2}-2 t_{2}^{2} t_{4} z_{1}\right)(12), \\
& t_{4} y_{1}(U y)=-t_{2}^{2} t_{4} z_{4}(12)+\left(t_{2}^{2} t_{1} z_{2}-t_{1}^{2} t_{2} z_{1}\right)(41), \\
& t_{1} y_{2}(U y)=-t_{4}^{2} t_{1} z_{1}(24)+\left(t_{4}^{2} t_{1} z_{4}-t_{2}^{2} t_{4} z_{2}\right)(12), \\
& t_{2} y_{4}(U y)=-t_{1}^{2} t_{2} z_{2}(41)+\left(t_{1}^{2} t_{2} z_{1}-t_{4}^{2} t_{1} z_{4}\right)(24),
\end{aligned}
$$

where $(i k)=t_{i} z_{k}-t_{k} z_{i}$. Each of the above quadrics vanishes for $z_{i}=t_{i}$, that is each conic on the right in (48) intersects the polar conic $C^{2}$ in the zero point. The three remaining variable intersections of these conics and the polar conic correspond to the intersection of the plane $\left(\alpha_{i} y_{i}\right)=0$ and the curve $C^{3}$ in $S_{3}$. Hence the system of quadrics (48) give a parametric representation of the curve $C^{3}$. To each $z$ in (48) there is a definite point $y$ in $S_{3}$ except at the base point of the system $z_{i}=t_{i}$. This representation can be put in a simpler form if we multiply the quadrics in (48) by $t_{i} t_{k}$, so that each quadric on the left has the common factor $t_{1} t_{2} t_{4}(U y)$, which may be dropped, leaving the parametric representation of the curve $C^{3}$ as follows:

$$
\begin{aligned}
& y_{0}=\rho\left[+2 t_{1}^{2} t_{3}^{3} z_{2}(41)+\left(-2 t_{1}^{2} t_{2}^{2} t_{4} z_{2}-2 t_{1} t_{2}^{3} t_{4} z_{1}\right)(12)\right] \\
& y_{1}=\rho\left[-t_{1} t_{2}^{3} t_{4} z_{4}(12)+\left(t_{1} t_{2}^{3} t_{4} z_{2}-t_{1}^{3} t_{2}^{2} z_{1}\right)(41)\right] \\
& y_{2}=\rho\left[-t_{2} t_{4}^{3} t_{1} z_{1}(24)+\left(t_{2} t_{4}^{3} t_{1} z_{4}-t_{2}^{3} t_{2}^{2} z_{2}\right)(12)\right] \\
& y_{4}=\rho\left[-t_{4} t_{1}^{3} t_{2} z_{2}(41)+\left(t_{4} t_{1}^{3} t_{2} z_{1}-t_{4}^{3} t_{1}^{2} z_{4}\right)(24)\right] .
\end{aligned}
$$

Hence the doubled $C^{2}$ in $S_{2}$ is mapped upon the doubled $C^{3}$ in the fixed $S_{3}$ by means of the equations in (49).

3. The locus of the zero point in $S_{3}$. In $S_{2}$ we find $K$ as the locus of the zero point. Each curve of the system $C^{2}$ has one such point, which generates $K$ by the variation of $\omega$. Each curve of the system $C^{3}$ has on it the zero point. What is the equation of its locus? Since $z_{i}=t_{i}$ is the base point of the mapping system which maps $C^{2}$ upon $C^{3}$, all the $y^{\prime}$ s vanish at this point, but as $z$ approaches $t$ the limiting position of the direction is that of the tangent to the polar conic $C^{2}$ at $z_{i}=t_{i}$. If the factors $(i k)$ in (49) are replaced by the coördinates of the tangent to the polar conic at the point $z_{i}=t_{i}$, and if we set $z_{i} \leq t_{i}$ in the other factors, the $y$ 's do not vanish, and become nonic functions of $t$ which have a common factor $t_{1} t_{2} t_{4}$. However, a much simpler way to get this parametric representation of the locus of the zero point in $S_{3}$ is to solve the bilinearforms $\phi_{01}^{\prime}$ $=\phi_{02}^{\prime}=\phi_{04}^{\prime}=0$ for $y_{i}$, and put $z_{i}=t_{i}$ in the result, from which the factor $t_{1} t_{2} t_{4}$ can be removed. These equations are:

$$
\begin{aligned}
& y_{0}=-14 t_{1}^{2} t_{2}^{2} t_{2}^{2}, \\
& y_{1}=t_{1}^{4} t_{4}^{2}-3 t_{1}^{3} t_{2}^{3}-5 t_{1} t_{2}^{2} t_{4}^{3}, \\
& y_{2}=t_{2}^{4} t_{1}^{2}-3 t_{2}^{3} t_{4}^{3}-5 t_{2} t_{4}^{2} t_{1}^{3}, \\
& y_{4}=t_{4}^{4} t_{2}^{2}-3 t_{4}^{3} t_{1}^{3}-5 t_{4} t_{1}^{2} t_{2}^{3} .
\end{aligned}
$$


These equations map the locus of the zero point in $S_{2}$ upon a locus in the space of the $y$ 's. The order of this locus is 18 , for a plane section $\left(U^{\prime} y\right)=0$ gives a sextic in $t$ which intersects $K$ in 24 points, but we find that this variable sextic and $K$ have 6 fixed intersections at the flex points $t_{i}=t_{k}=0$ and consequently 18 variable ones. Hence the locus of the zero point in $S_{3}$ is a curve of order 18 and will be denoted by $C^{18}$.

It has already been pointed out that the order of the group of the $y$ 's is double the order of the group of the $z$ 's and that to express a form in $y$ and $z$ covariantly its points and planes in $S_{3}$ must appear squared. This $C^{18}$ can evidently be represented covariantly if we take the 10 quadratic combinations of the $y$ 's from the equations (50) from which we can eliminate the factor $t_{1} t_{2} t_{4}$ and thereby eliminate the fixed intersections each taken twice, and if we take in primed variables the corresponding quadratic combinations of the $U$ 's as the coefficients of these quadratic combinations of the $y$ 's. This form is

$$
f\left(t^{\prime 3}, t^{9}\right)=0,
$$

and is of the third order in $t^{\prime}$, and of the ninth order in $t$. The number of variable points in which this nonic intersects $K$ is 36 , which is double the order of $C^{18}$, since its points appear squared in (51). If $t=t^{\prime}$ in (51) we find a form of order 12 which is $K^{3}+16 H^{2}$, where $H$ is the Hessian of $K$. We can then say that the form (51) is the third polar of $K^{3}+16 H^{2}$ plus covariant terms containing the line coördinates $t t^{\prime}$. To obtain these further terms one would make use of the complete system of invariants and covariants of $K$ which has been calculated and tabulated by Gordan.*

4. Summary. The results obtained may be briefly summarized. The well known elliptic modular functions associated with the elliptic norm curve $E^{7}$ and the algebraic relations connecting them have been readily found from the geometric point of view. The system of contact cubics in (37), the coordinates of the modular lines $L_{m}$ and $L_{m}^{\prime}$ and the parametric representation of the locus of the zero point in $S_{3}$ are new types of functions. The system of modular functions $B \gamma$ (in Klein's notation) $\dagger$ which define a curve of order 14 has not been found.

If a pair of points in the involution on the curve $E^{7}$ is isolated, the quadratic irrationality associated with the curve $E^{7}$ is obtained. This irrationality can be obtained from the system $y_{i}$ in (44). If we substitute the values of these $y_{i}$ in any of the quadrics (19) (except those bilinear in $y$ and $z$ ), $\rho$ is obtained as the square root of the reciprocal of a conic $g\left(t^{6}, z^{2}\right)$. This conic has the form

$$
\begin{gathered}
g\left(t^{6}, z^{2}\right)=z_{1}^{2}\left(2 t_{1} t_{2}^{4} t_{4}+t_{1}^{2} t_{2} t_{4}^{3}\right)+z_{2}^{2}\left(2 t_{1} t_{2} t_{4}^{4}\right)+z_{4}^{2}\left(t_{1}^{4} t_{2} t_{4}\right)+z_{1} z_{2}\left(t_{1}^{2} t_{2}^{3} t_{4}\right. \\
\left.-2 t_{1}^{3} t_{4}^{3}\right)+z_{2} z_{4}\left(-4 t_{1}^{3} t_{2}^{3}-t_{2}^{5} t_{4}\right)-z_{1} z_{4}\left(3 t_{2}^{3} t_{4}^{3}\right)
\end{gathered}
$$

* M a thematis che An nal e n, vol. 17 (1880), pp. 217, 359.

† See K. F., vol. 2, p. 396-397. 
and constitutes the part in $z$ of a quadric on the curve $E^{7}$ whose part in $y$ is the square of the half period plane (47).* We can now write down the parametric representation of the curve $E^{7}$. It is

$$
\begin{aligned}
& y_{0}^{\prime}=+2 t_{1}^{2} t_{2}^{3} z_{2}(41)-\left(2 t_{1}^{2} t_{2}^{2} t_{4} z_{2}+2 t_{1} t_{2}^{3} t_{4} z_{1}\right)(12), \\
& y_{1}^{\prime}=-t_{1} t_{2}^{3} t_{4} z_{4}(12)+\left(t_{1} t_{2}^{3} t_{4} z_{2}-t_{1}^{3} t_{2}^{2} z_{1}\right)(41) \text {, } \\
& y_{2}^{\prime}=-t_{2} t_{4}^{3} t_{1} z_{1}(24)+\left(t_{2} t_{4}^{3} t_{1} z_{4}-t_{2}^{3} t_{4}^{2} z_{2}\right)(12) \text {, } \\
& y_{4}^{\prime}=-t_{4} t_{1}^{3} t_{2} z_{2}(41)+\left(t_{4} t_{1}^{3} t_{2} z_{1}-t_{4}^{3} t_{1}^{2} z_{4}\right)(24) \text {, } \\
& z_{1}^{\prime}=z_{1} t_{1} t_{2} t_{4} \sqrt{g\left(t^{6}, z^{2}\right)} \text {, } \\
& z_{2}^{\prime}=z_{2} t_{1} t_{2} t_{4} \sqrt{g\left(t^{6}, z^{2}\right.}, \\
& z_{4}^{\prime}=z_{4} t_{1} t_{2} t_{4} \sqrt{g\left(t^{6}, z^{2}\right)} \text {. }
\end{aligned}
$$

If $t$ is on $K$ the above system maps the doubled $C^{2}$ upon the $E^{7}$. It should be noted that the $y^{\prime}$ 's vanish for $z_{i}=t_{i}$ and the $z$ 's vanish when $z$ is on a half period point. $†$

* Professor Sharpe of Cornell pointed out this fact to me, as well as a method of eliminating an extraneous factor $t_{1} h_{2} l_{4}$ from the parametric representation of the curve $E^{7}$. I append the method in a foot note at the end of the paper.

+ All the terms in $y_{i}^{\prime}$ contain the factor $t_{1} t_{2} t_{4}$ except one term in $y_{0}^{\prime}$ and this term contains a $z_{4}$. If we now find the intersection of the pencil of lines through the point $t, \lambda_{1}(42)+\lambda_{2}(14)$ $=0$ and the polar conic $C^{2}$, we get the following values for $z_{i}$ :

$$
\begin{aligned}
& z_{1}=\lambda_{1}^{2}\left(-2 t_{1}^{2} t_{2}-t_{4}^{3}\right)+\lambda_{2}^{2} t_{1} t_{2} t_{4}-3 t_{2}^{2}+t_{4} \lambda_{1} \lambda_{2}, \\
& z_{2}=\lambda_{1}^{2} t_{1} t_{2}^{2}+\lambda_{2}^{2}\left(-2 t_{2}^{2} t_{4}-t_{1}^{3}\right)-\left(2 t_{1}^{2} t_{2}+t_{4}^{3}\right) \lambda_{1} \lambda_{2}, \\
& z_{4}=\lambda_{1}^{2} t_{1} t_{2} t_{4}+\lambda_{2}^{2} t_{2} t_{4}^{2} t_{1}^{2}+t_{4} \lambda_{1} \lambda_{2} .
\end{aligned}
$$

Hence when these values are put in (53) the factor $t_{1} t_{2} t_{8}$ can be removed.

UNIVERSITY OF ILLINOIS,

URBANA, ILL. 
VI'TA

Born at Mayo, Ky., August 10, 1859, son of 'Thomas Clinton and Margaret Wheeler-Woods. Received his elementary education in the public school in Hopewell District, Mercer County, Ky. Entered the academy connected with Georgetown College at Georgetown, Ky., in 1908 and graduated from Georgetown College in 1914 with A.B. degree. Spent the summer of 1914 as a graduate student in the University of Chicago. Held an Assistantship in Mathematics in the University of Maine during the year 1914-15, Instructor, 1915-17. Received A.M. degree from the University of Maine in 1916. Was an Assistant in Mathematics in the University of Illinois during the years 1917-20. 
UNIVERSITY OF CALIFORNIA LIBRARY, BERKELEY

THIS BOOK IS DUE ON THE LAST DATE STAMPED BELOW

Books not retnrned on time are subject to a fine of $50 \mathrm{c}$ per volume after the third day to $\$ 1.00$ per volnme after the airth der. demsnd pay be renewed if application is mode not expiration of loan period.

SEP 121924 


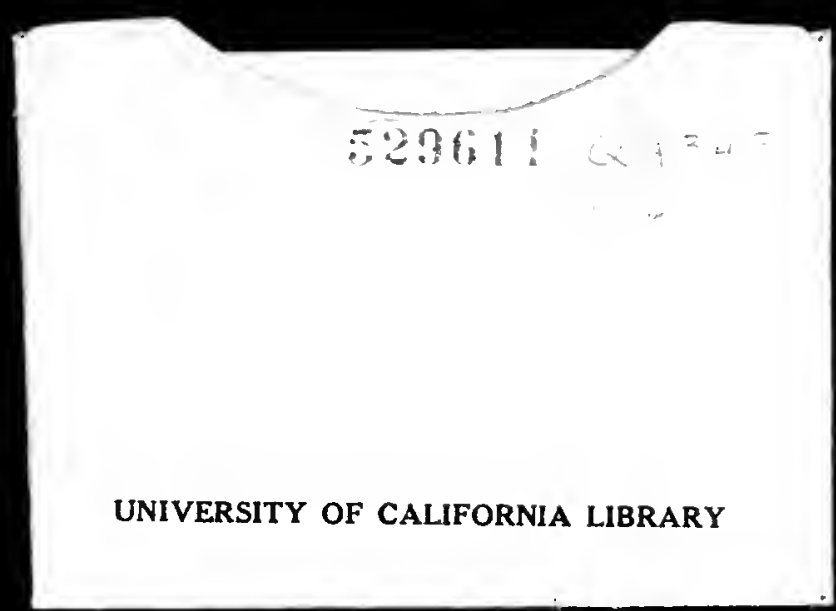


